\title{
Mathematical Modeling and Analysis of Nonlinear Enzyme Catalyzed Reaction Processes
}

\author{
D. Mary Celin Sharmila, T. Praveen, and L. Rajendran \\ Department of Mathematics, The Madura College, Madurai 625 011, Tamil Nadu, India \\ Correspondence should be addressed to L. Rajendran; raj_sms@rediffmail.com
}

Received 20 March 2013; Accepted 28 October 2013

Academic Editors: A. M. Lamsabhi, A. Stavrakoudis, and B. M. Wong

Copyright ( 2013 D. Mary Celin Sharmila et al. This is an open access article distributed under the Creative Commons Attribution License, which permits unrestricted use, distribution, and reproduction in any medium, provided the original work is properly cited.

A mathematical model for the nonlinear enzymatic reaction process is discussed. An approximate analytical expression of concentrations of substrate, enzyme, and free enzyme-product is obtained using homotopy perturbation method (HPM). The main objective is to propose an analytical solution, which does not require small parameters and avoid linearization and physically unrealistic assumptions. Theoretical results obtained can be used to analyze the effect of different parameters. Satisfactory agreement is obtained in the comparison of approximate analytical solution and numerical simulation.

\section{Introduction}

The importance of biocatalytic processes and reactions for organic synthesis and the pharmaceutical food and cosmetics industry has been constantly growing during the last few years $[1,2]$. From a synthetic point of view, enzymes are highly efficient catalysts for an extremely broad palette of reactions [3]. Enzymes of one type, but from different origins, are specialized for substrates, positions in substrates, and products [4]. Enzyme reactions do not follow the law of mass action directly. The rate of the reaction only increases to a certain extent as the concentration of substrate increases. The maximum reaction rate is reached at high substrate concentration due to enzyme saturation. This is in contrast to the law of mass action that states that the reaction rate increases as the concentration of substrate increases [5]. Various simplified analytical models have been developed over the last 20 years. In brief, the analysis involves the construction and solution of reaction/diffusion differential equations, resulting in the development of approximate analytical expressions for $[6,7]$ nonlinear enzyme catalyzed reaction processes.

The simplest model that explains the kinetic behaviour of enzyme reactions is the classic 1913 model of Michaelis and Menten [8] which is widely used in biochemistry for many types of enzymes. The Michaelis-Menten model is based on the assumption that the enzyme binds the substrate to form an intermediate complex which then dissociates to form the final product and release the enzyme in its original form. The schematic representation of this two-step process is given by

$$
\mathrm{E}+\mathrm{S} \underset{k_{-1}}{\stackrel{k_{1}}{\leftrightarrow}} \mathrm{C} \stackrel{k_{2}}{\longrightarrow} \mathrm{E}+\mathrm{P},
$$

where $k_{1}, k_{-1}$, and $k_{2}$ are constant parameters associated with the rates of the reaction. Note that it is generally assumed that the second step of the reaction equation (1) is irreversible. In reality, this is not always the case. Typically, reaction rates are measured under the condition that the product is continually removed, which prevents the reverse reaction of the second step from occurring effectively.

In this paper we have derived an expression for concentration of substrate, enzyme-substrate, and free enzymeproduct with nonmechanism based enzyme inactivation, in terms of dimensionless reaction diffusion parameters $\varepsilon, \lambda_{1}$, $\lambda_{2}$, and $\lambda_{3}$ using homotopy perturbation method (HPM). Comparative study of the same with numerical simulation is presented.

\section{Mathematical Formulation of the Problems}

If a small amount of enzyme is used and all but one substrate is kept constant, then the rate of the enzymatically catalyzed 
reaction depends on the substrate concentration and initial rate as in the equation $v=v_{\max }[\mathrm{S}] /\left(\mathrm{K}_{M}+[\mathrm{S}]\right)$, given by [9], where $K_{M}$ is the Michaelis constant: $K_{M}=\left(k_{-1}+k_{2}\right) / k_{1}$. The typical notation of the enzyme catalyzed reaction with one substrate can be given as [10]

$$
\mathrm{A}+\mathrm{E} \underset{k_{2}}{\stackrel{k_{1}}{\leftrightarrow}} \mathrm{X} \underset{k_{4}}{\stackrel{k_{3}}{\leftrightarrow}} \mathrm{P}+\mathrm{E}
$$

where $\mathrm{A}$ is substrate, $\mathrm{E}$ is enzyme, $\mathrm{X}$ is enzyme-substrate complex, and $\mathrm{P}$ is free enzyme product. The kinetic equations consist of

$$
\begin{gathered}
\frac{d[\mathrm{~A}]}{d t}=k_{2}[\mathrm{X}]-k_{1}[\mathrm{~A}][\mathrm{E}] \\
\frac{d[\mathrm{E}]}{d t}=\left(k_{2}+k_{3}\right)[\mathrm{X}]-\left(k_{1}[\mathrm{~A}]+k_{4}[\mathrm{P}]\right)[\mathrm{E}] \\
\frac{d[\mathrm{P}]}{d t}=k_{3}[\mathrm{X}]-k_{4}[\mathrm{P}][\mathrm{E}]
\end{gathered}
$$

with a conservation relation given in [6]:

$$
[\mathrm{E}]+[\mathrm{X}]=[\mathrm{E}]_{\text {total }} \text {. }
$$

It is obvious that the derivative of a substrate with respect to time gives the rate. Thus, the rate is a function of compounds [V], [A] (intracellular and extracellular), enzyme concentrations $\mathrm{E}$ and kinetic parameter $k$. However, the enzyme concentration is hidden in the kinetic constants in the parameter vector $k$; herewith, we can write $v$ as a function of [V], [A], and [P]; that is, $v=v([\mathrm{~A}],[\mathrm{V}], k)[11]$.

The more general form of $(3 a)-(3 c)$ can be written in the form of

$$
\begin{gathered}
\frac{d[\mathrm{~A}]}{d t}=v_{2}-v_{1}, \\
\frac{d[\mathrm{E}]}{d t}=v_{2}+v_{3}-v_{1}-v_{4}, \\
\frac{d[\mathrm{X}]}{d t}=v_{1}+v_{4}-v_{2}-v_{3}, \\
\frac{d[\mathrm{P}]}{d t}=v_{3}-v_{4} .
\end{gathered}
$$

The form of rate equations is as follows:

$$
\begin{aligned}
& v_{1}=k_{1}[\mathrm{~A}][\mathrm{E}], \quad v_{2}=k_{2}[\mathrm{X}], \\
& v_{3}=k_{3}[\mathrm{X}], \quad v_{4}=k_{4}[\mathrm{P}][\mathrm{E}] .
\end{aligned}
$$

The initial condition at $t=0$ are as follows:

$$
[\mathrm{A}]=\mathrm{A}_{0}, \quad[\mathrm{E}]=\mathrm{E}_{0}, \quad[\mathrm{X}]=0, \quad[\mathrm{P}]=0 .
$$

The concentration of the reactants in $(5 a)-(5 d)$ is denoted by lower case letters

$$
s=[\mathrm{A}], \quad e=[\mathrm{E}], \quad c=[\mathrm{X}], \quad p=[\mathrm{P}],
$$

The law of mass action leads to the system of following nonlinear kinetic equations [12]:

$$
\begin{gathered}
\frac{d s}{d t}=-k_{1} s e+k_{2} c \\
\frac{d e}{d t}=-k_{1} s e+k_{2} c+k_{3} c-k_{4} p e \\
\frac{d p}{d t}=k_{3} c-k_{4} p e \\
\frac{d c}{d t}=k_{1} s e-k_{2} c-k_{3} c+k_{4} p e
\end{gathered}
$$

with the boundary conditions being

$$
s(0)=s_{0}, \quad e(0)=e_{0}, \quad p(0)=0, \quad c(0)=0 .
$$

Adding (9b) and (9c), we get

$$
\frac{d e}{d t}+\frac{d c}{d t}=0
$$

Using the initial conditions (10) we obtain

$$
e(t)+c(t)=e_{0} .
$$

With this, the system of ordinary differential equations reduces to the following three differential equations:

$$
\begin{gathered}
\frac{d s}{d t}=-k_{1} s\left(e_{0}-c\right)+k_{2} c, \\
\frac{d c}{d t}=k_{1} s\left(e_{0}-c\right)-\left(k_{2}+k_{3}\right) c+k_{4} p\left(e_{0}-c\right), \\
\frac{d p}{d t}=k_{3} c-k_{4} p\left(e_{0}-c\right) .
\end{gathered}
$$

With initial conditions $s(0)=s_{0}, c(0)=0$, and $p(0)=0$. By introducing the following set of nondimensional variables and parameters,

$$
\begin{aligned}
& \tau=\frac{k_{1} e_{0} t}{\varepsilon}, \quad u(\tau)=\frac{s(t)}{s_{0}}, \quad v(\tau)=\frac{c(t)}{s_{0}}, \\
& w(\tau)=\frac{p(t)}{s_{0}}, \quad \lambda_{1}=\frac{k_{2}}{k_{1} s_{0}}, \quad \lambda_{2}=\frac{k_{3}}{k_{1} s_{0}}, \\
& \lambda_{3}=\frac{k_{4}}{k_{1}}, \quad \varepsilon=\frac{e_{0}}{s_{0}}, \quad \eta=\lambda_{1}+\lambda_{2},
\end{aligned}
$$

the system of (13a)-(13c) and the initial conditions (10) can be represented in dimensionless form as follows:

$$
\frac{d u}{d \tau}=-\varepsilon u+u v+\lambda_{1} v
$$

$$
\begin{gathered}
\frac{d v}{d \tau}=\varepsilon u-\eta v+\lambda_{3} \varepsilon w-u v-\lambda_{3} v, \\
\frac{d w}{d \tau}=\lambda_{2} v-\lambda_{3} \varepsilon w+\lambda_{3} v w,
\end{gathered}
$$

with

$$
u(0)=1, \quad v(0)=0, \quad w(0)=0 .
$$




\section{Implementation of the HPM}

We indicate how (33)-(35) in this paper are derived. To find the solution of (14)-(16), we first construct a homotopy as follows:

$$
\begin{aligned}
& (1-p)\left[\frac{d u}{d \tau}+\varepsilon u\right]+p\left[\frac{d u}{d \tau}+\varepsilon u-u v-\lambda_{1} v\right]=0 \\
& (1-p)\left(\frac{d v}{d \tau}+\eta v\right) \\
& +p\left(\frac{d v}{d \tau}+\eta v-\varepsilon u-\lambda_{3} \varepsilon w+u v+\lambda_{3} v w\right)=0 \\
& (1-p)\left(\frac{d w}{d \tau}+\lambda_{3} \varepsilon w\right)+p\left(\frac{d w}{d \tau}+\lambda_{3} \varepsilon w-\lambda_{2} v-\lambda_{3} v w\right)=0
\end{aligned}
$$

And the initial approximations are as follows:

$$
u(0)=1, \quad v(0)=0, \quad w(0)=0 .
$$

Approximate solutions of (33)-(35) are

$$
\begin{gathered}
u=u_{0}+p u_{1}+p^{2} u_{2}+p^{3} u_{3}+\cdots \\
v=v_{0}+p v_{1}+p^{2} v_{2}+p^{3} v_{3}+\cdots \\
w=w_{0}+p w_{1}+p^{2} w_{2}+p^{3} w_{3}+\cdots
\end{gathered}
$$

Substituting (23)-(25) into (19)-(21), respectively, and comparing the coefficients of like powers of $p$, we can obtain the following differential equations for the concentration of substrate:

$$
\begin{gathered}
p^{0}: \frac{d u_{0}}{d \tau}+\varepsilon u_{0}=0, \\
p^{1}: \frac{d u_{1}}{d \tau}+\varepsilon u_{1}-u_{0} v_{0}-\lambda_{1} v_{0}=0, \\
p^{2}: \frac{d u_{2}}{d \tau}+\varepsilon u_{2}-\left(u_{0} v_{1}+u_{1} v_{0}\right)-\lambda_{1} v_{1}=0 .
\end{gathered}
$$

For enzyme substrate concentration $v$,

$$
\begin{gathered}
p^{0}: \frac{d v_{0}}{d \tau}+\eta v_{0}=0, \\
p^{1}: \frac{d v_{1}}{d \tau}+\eta v_{1}-\varepsilon u_{0}-\lambda_{3} \varepsilon w_{0}+u_{0} v_{0}+\lambda_{3} v_{0} w_{0}=0 \\
p^{2}: \frac{d v_{2}}{d \tau}+\eta v_{2}-\varepsilon u_{1}-\lambda_{3} \varepsilon w_{1}+u_{0} v_{1} \\
+u_{1} v_{0}+\lambda_{3}\left(v_{0} w_{1}+v_{1} w_{0}\right)=0 .
\end{gathered}
$$

For product concentration $w$

$$
\begin{gathered}
p^{0}: \frac{d w_{0}}{d \tau}+\lambda_{3} \varepsilon w_{0}=0, \\
p^{1}: \frac{d w_{1}}{d \tau}+\lambda_{3} \varepsilon w_{1}-\lambda_{2} v_{0}-\lambda_{3} v_{0} w_{0}=0, \\
p^{2}: \frac{d w_{2}}{d \tau}+\lambda_{3} \varepsilon w_{2}-\lambda_{2} v_{1}-\lambda_{3}\left(v_{0} w_{1}+v_{1} w_{0}\right)=0,
\end{gathered}
$$

$$
p^{3}: \frac{d w_{2}}{d \tau}+\lambda_{3} \varepsilon w_{3}-\lambda_{2} v_{2}-\lambda_{3}\left(v_{0} w_{2}+v_{1} w_{1}+v_{2} w_{0}\right)=0
$$

Solving (26)-(28), and using the boundary conditions (22), we can find the following results:

$$
\begin{aligned}
& u_{0}(\tau)=e^{-\varepsilon \tau}, \\
& u_{1}(\tau)=0 \text {, } \\
& u_{2}(\tau)=\frac{1}{(\eta-\varepsilon)}\left[-e^{-2 \varepsilon \tau}+e^{-\varepsilon \tau}\right]+\frac{\varepsilon}{\eta(\eta-\varepsilon)}\left[e^{-(\eta+\varepsilon)}-e^{-\varepsilon t}\right] \\
& +\frac{\lambda_{1} \varepsilon \tau e^{-\varepsilon \tau}}{(\eta-\varepsilon)}+\frac{\lambda_{1} \varepsilon}{(\eta-\varepsilon)}\left[e^{-\eta \tau}-e^{-\varepsilon \tau}\right] \text {, } \\
& v_{0}(\tau)=0 \text {, } \\
& v_{1}(\tau)=\frac{\varepsilon}{\eta-\varepsilon}\left[e^{-\varepsilon \tau}-e^{-\eta \tau}\right] \\
& v_{2}(\tau)=\frac{\varepsilon\left[e^{-\eta \tau}-e^{-2 \varepsilon \tau}\right]}{(\eta-\varepsilon)(2 \varepsilon-\eta)}+\frac{\left[e^{-(\varepsilon+\eta) \tau}-e^{-\eta \tau}\right]}{(\eta-\varepsilon)}, \\
& w_{0}(\tau)=0 \text {, } \\
& w_{1}(\tau)=0, \\
& w_{2}(\tau)=\frac{\lambda_{2} \varepsilon}{(\eta-\varepsilon)}\left[\frac{\left(e^{-\varepsilon \tau}-e^{-\lambda_{3} \varepsilon \tau}\right)}{\left(\lambda_{3}-1\right) \varepsilon}-\frac{\left(e^{-\eta \tau}-e^{-\lambda_{3} \varepsilon \tau}\right)}{\left(\lambda_{2} \varepsilon-\eta\right)}\right], \\
& w_{3}(\tau)=\frac{\lambda_{2}\left[e^{-2 \varepsilon \tau}-e^{-\lambda_{3} \varepsilon \tau}\right]}{(\varepsilon-\eta)(\eta-2 \varepsilon)\left(\lambda_{3}-2\right)}-\frac{\lambda_{2}\left[e^{-(\varepsilon+\eta) \tau}-e^{-\lambda_{3} \varepsilon \tau}\right]}{(\varepsilon-\eta)\left(\lambda_{3}-\varepsilon-\eta\right)} \\
& -\frac{\lambda_{2} \varepsilon\left[e^{-\eta \tau}-e^{-\lambda_{3} \varepsilon \tau}\right]}{(\varepsilon-\eta)(\eta-2 \varepsilon)\left(\lambda_{3} \varepsilon-\eta\right)}+\frac{\lambda_{2}\left[e^{-\eta \tau}-e^{-\lambda_{3} \varepsilon \tau}\right]}{(\varepsilon-\eta)\left(\lambda_{3} \varepsilon-\eta\right)} \text {. }
\end{aligned}
$$

According to the HPM, we can conclude that

$$
\begin{gathered}
u(\tau)=\lim _{p \rightarrow 1} u(\tau)=u_{0}+u_{1}+u_{2}, \\
v(\tau)=\lim _{p \rightarrow 1} v(\tau)=v_{0}+v_{1}+v_{2}, \\
w(\tau)=\lim _{p \rightarrow 1} w(\tau)=w_{0}+w_{1}+w_{2}+w_{3} .
\end{gathered}
$$

Substitute (29) in (30)-(31) we obtain (33)-(35) in the text.

\section{Analytical Solution of Substrate, Enzyme, Enzyme-Substrate Complex, and Free Enzyme Product Using HPM}

Non-linear phenomena play a crucial role in applied mathematics and chemistry. Construction of particular exact solutions for these equations remains an important problem. Finding exact solutions that have a physical, chemical, or biological interpretation is of fundamental importance. The investigation of exact solution of non-linear equation is 
interesting and important. In the past, many authors mainly had paid attention to study solution of non-linear equations by using various methods, variational iteration method [13], and homotopy perturbation method [14-17].

The homotopy perturbation method has been extensively worked out over a number of years by numerous authors. The idea has been used to solve nonlinear boundary value problems [15], integral equations [18-20], Klein-Gordon and Sine-Gordon equations [21], Emden-Flower type equations [22], and many other problems. This wide variety of applications shows the power of the HPM to solve functional equations. The HPM is unique in its applicability, accuracy and efficiency. The HPM [23] uses the imbedding parameter $p$ as a small parameter, and only a few iterations are needed to search for an asymptotic solution. Using this method, we can obtain the following solution to (14)-(16) (see the appendix):

$$
\begin{aligned}
u(\tau)= & e^{-\varepsilon \tau}+\frac{\left[-e^{-2 \varepsilon \tau}+e^{-\varepsilon \tau}\right]}{(\eta-\varepsilon)}+\frac{\varepsilon\left[e^{-(\eta+\varepsilon)}-e^{-\varepsilon \tau}\right]}{\eta(\eta-\varepsilon)} \\
& +\frac{\lambda_{1} \varepsilon \tau e^{-\varepsilon \tau}}{(\eta-\varepsilon)}+\frac{\lambda_{1} \varepsilon\left[e^{-\eta \tau}-e^{-\varepsilon \tau}\right]}{(\eta-\varepsilon)}, \\
v(\tau)= & \frac{\varepsilon}{\eta-\varepsilon}\left[e^{-\varepsilon \tau}-e^{-\eta \tau}\right]+\frac{\varepsilon\left[e^{-\eta \tau}-e^{-2 \varepsilon \tau}\right]}{(\eta-\varepsilon)(2 \varepsilon-\eta)} \\
& +\frac{\left[e^{-(\varepsilon+\eta) \tau}-e^{-\eta \tau}\right]}{(\eta-\varepsilon)}, \\
w(\tau)= & \frac{\lambda_{2} \varepsilon}{(\eta-\varepsilon)}\left[\frac{\left(e^{-\varepsilon \tau}-e^{-\lambda_{3} \varepsilon \tau}\right)}{\left(\lambda_{3}-1\right) \varepsilon}-\frac{\left(e^{-\eta \tau}-e^{-\lambda_{3} \varepsilon \tau}\right)}{\left(\lambda_{2} \varepsilon-\eta\right)}\right] \\
& +\frac{\lambda_{2}\left[e^{-2 \varepsilon \tau}-e^{-\lambda_{3} \varepsilon \tau}\right]}{(\varepsilon-\eta)(\eta-2 \varepsilon)\left(\lambda_{3}-2\right)}-\frac{\lambda_{2}\left[e^{-(\varepsilon+\eta) \tau}-e^{-\lambda_{3} \varepsilon \tau}\right]}{(\varepsilon-\eta)\left(\lambda_{3}-\varepsilon-\eta\right)} \\
& -\frac{\lambda_{2} \varepsilon\left[e^{-\eta \tau}-e^{-\lambda_{3} \varepsilon \tau}\right]}{(\varepsilon-\eta)(\eta-2 \varepsilon)\left(\lambda_{3} \varepsilon-\eta\right)}+\frac{\lambda_{2}\left[e^{-\eta \tau}-e^{-\lambda_{3} \varepsilon \tau}\right]}{(\varepsilon-\eta)\left(\lambda_{3} \varepsilon-\eta\right)}
\end{aligned}
$$

Equations (33)-(35) represent the analytical expression of the dimensionless substrate concentration $u(\tau)$, dimensionless enzyme-substrate concentration $v(\tau)$, and dimensionless free enzyme product concentration $w(\tau)$ for all values of parameters $\varepsilon, \lambda_{1}, \lambda_{2}$ and $\lambda_{3}$. For steady condition, the differential equations (17a)-(17c) become as follows:

$$
\begin{gathered}
-\varepsilon u+u v+\lambda_{1} v=0, \\
\varepsilon u-\eta v+\lambda_{3} \varepsilon w-u v-\lambda_{3} v=0, \\
\lambda_{2} v-\lambda_{3} \varepsilon w+\lambda_{3} v w=0 .
\end{gathered}
$$

Solving the above equations, we can obtain the concentrations of substrate $u$, enzyme substrate complex $v$, and product free enzyme $w$ as follows: $u=0, v=0, w=0$. When $\tau$ tends to infinity, the analytical expression corresponding to the substrate concentration $u$, enzyme substrate

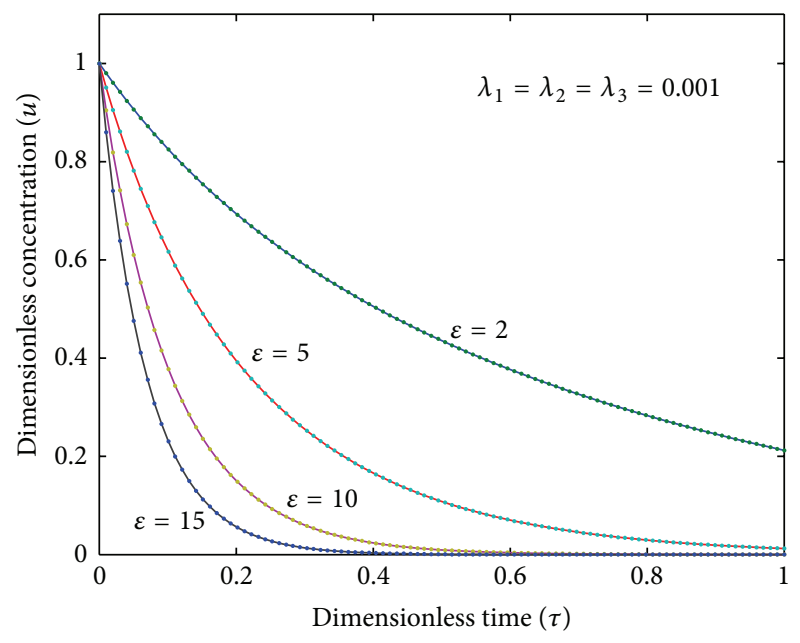

(a)

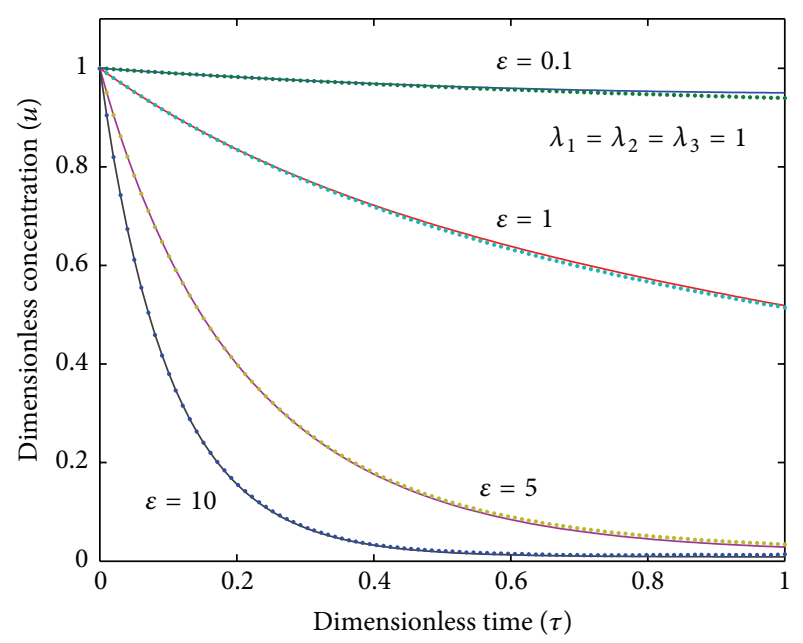

(b)

FIGURE 1: Profile of the normalized concentrations of substrate $u$ is calculated using (33) for various values of dimensionless parameters $\varepsilon, \lambda_{1}, \lambda_{2}$, and $\lambda_{3}$. Solid line: (33); dotted line: numerical simulation.

concentration $v$ and free enzyme product concentration $w$ from (33)-(35) confirm the validity of mathematical analysis.

\section{Results and Discussion}

The substrate concentration versus time is plotted in Figures 1 and 2 using (33). From Figures 1(a) and 1(b), it is observed that the dimensionless substrate concentration $u$ decreases. When parameters $\lambda_{1}=\lambda_{2}=\lambda_{3}=0.001$, the dimensionless substrate concentration $u$ gradually decreases as the value of parameter $\varepsilon$ increases and reaches the steady state when $\tau \geq 0.5$. When $\lambda_{1}=\lambda_{2}=\lambda_{3}=1$, the concentration decreases rapidly as $\varepsilon$ increases. $u \approx 1$ when $\varepsilon \leq 0.1$. In Figure 2 , it is inferred that the concentration decreases as dimensionless time $\tau$ increases and reaches its minimum when $\tau=1$. The graph is shown for various values of $\lambda_{1}$ when $\lambda_{2}=\lambda_{3}=$ $0.001, \varepsilon=1$. 


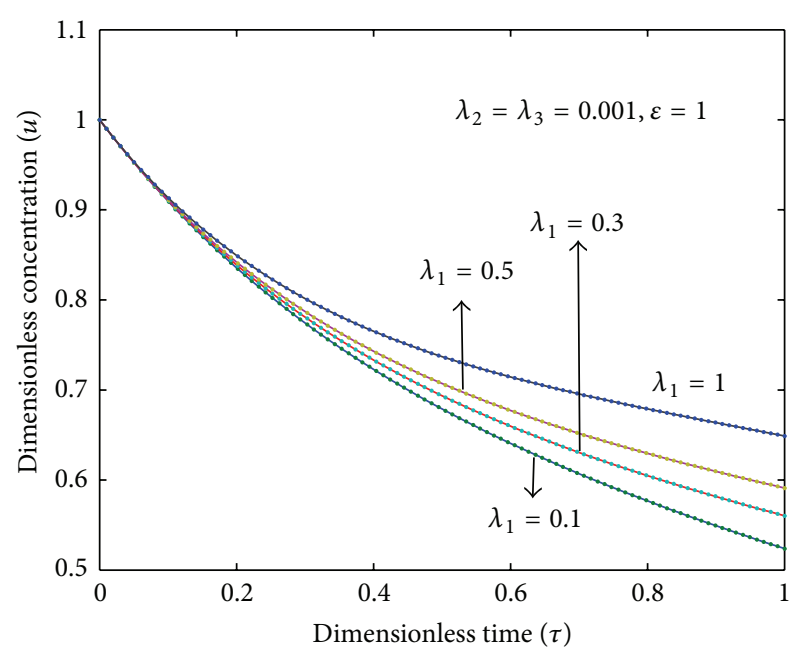

FIgURE 2: Profile of the normalized concentrations of substrate $u$ is calculated using (33) for various values of dimensionless parameter $\lambda_{1}$. Solid line: (33); dotted line: numerical simulation.

Figures 3 and 4 are plotted using (34), dimensionless time $\tau$ as abscissa, and enzyme concentration $v$ as ordinate. From Figures 3(a) and 3(b), it is observed that the enzyme concentration increases when $\tau \leq 0.2$ and reaches the steady state value when $\tau>0.2$. The graph is shown for various values of the parameter $\varepsilon$, when $\lambda_{1}=\lambda_{2}=\lambda_{3}=0.001$ and 0.01 . From Figure 4, it is inferred that enzyme concentration $v$ reaches its maximum between the time $0.03-0.09$, and reaches the steady state when $\tau \geq 0.5$.

Figures 5 and 6 shows the free enzyme product concentration versus time for various values of parameter $\lambda$ using (35). From Figures 5(a)-5(b), it is inferred that product concentration $w$ increases very slowly as the time increases. The graphs are shown for various values of the parameter $\varepsilon$, when $\lambda_{1}=\lambda_{2}=\lambda_{3}=0.001$ and 0.01 . In Figure 6, it is noted that the product concentration increases slowly and reaches the steady state at $\tau \geq 0.3$. Profile of dimensionless concentrations $u, v$, and $w$ versus the dimensionless time $\tau$ using (33), (34), and (35) for the fixed values of the parameters is plotted in Figure 7. From this figure, it is inferred that the concentration of substrate decreases, whereas the concentration of enzyme increases. But for all time the concentration of free enzyme-product have at most constant value.

\section{Conclusion}

Approximate analytical solutions to the system of nonlinear reaction equations in enzyme reaction mechanism are presented using homotopy perturbation method. A simple, straight forward, and a new method of estimating the concentrations of substrate, enzyme-substrate, and product are derived. This solution procedure can be easily extended to all kinds of system of coupled non-linear equations with various complex boundary conditions in enzyme-substrate nonlinear reaction diffusion processes.

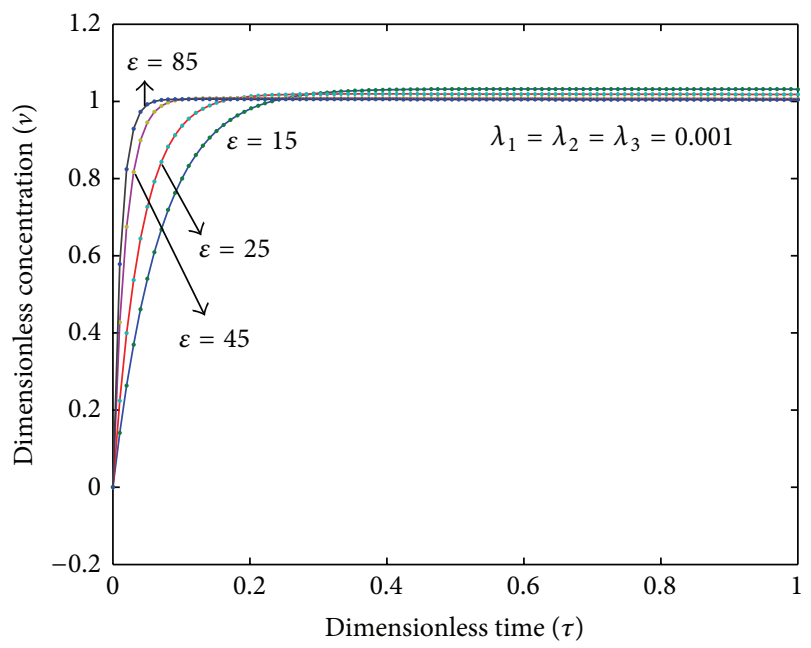

(a)

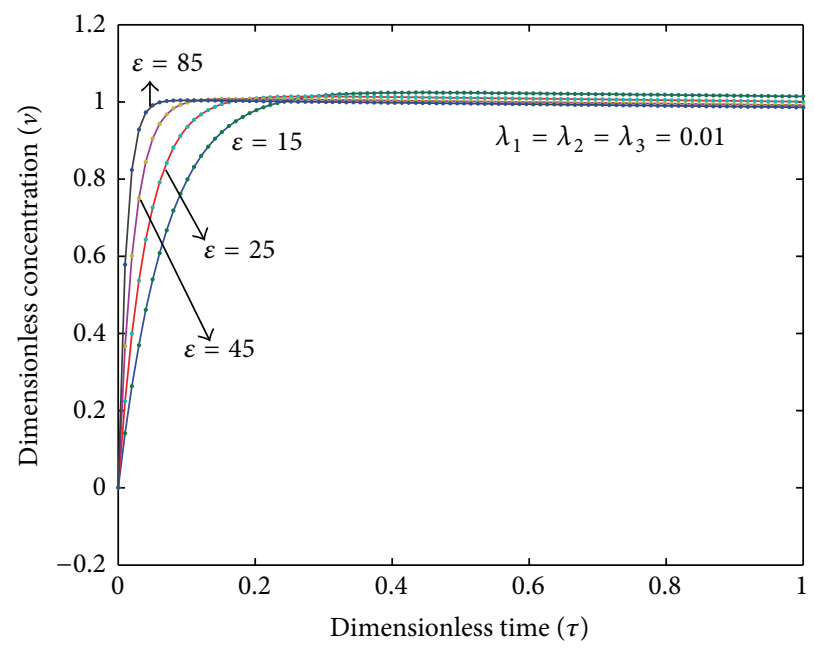

(b)

FIgURE 3: Profile of dimensionless enzyme concentration $v$ is calculated using (34) for various values of dimensionless parameters $\varepsilon, \lambda_{1}, \lambda_{2}$ and $\lambda_{3}$. Solid line: (34); dotted line: numerical simulation.

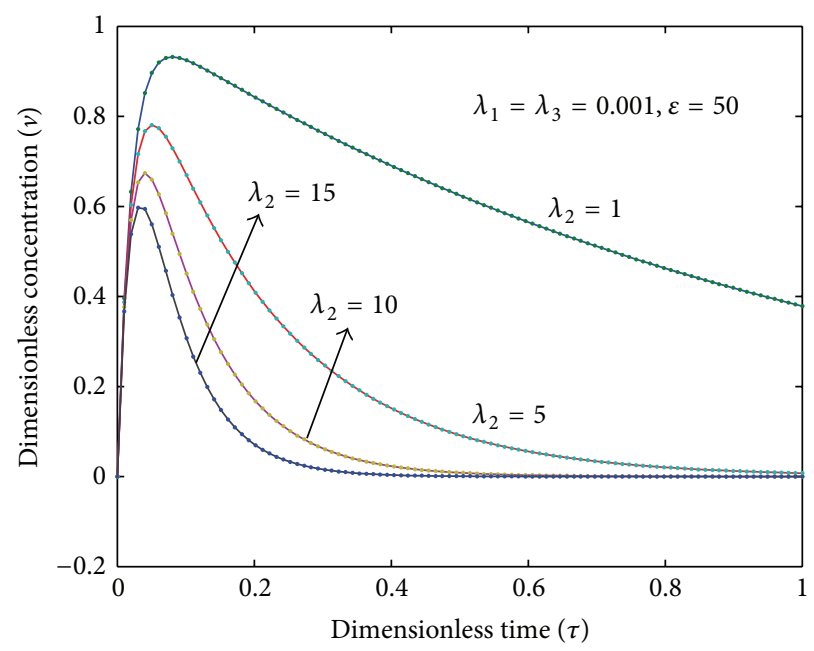

Figure 4: Profile of dimensionless enzyme concentration $v$ is calculated using (34) for various values of dimensionless parameter $\lambda_{2}$. Solid line: (34); dotted line: numerical simulation. 


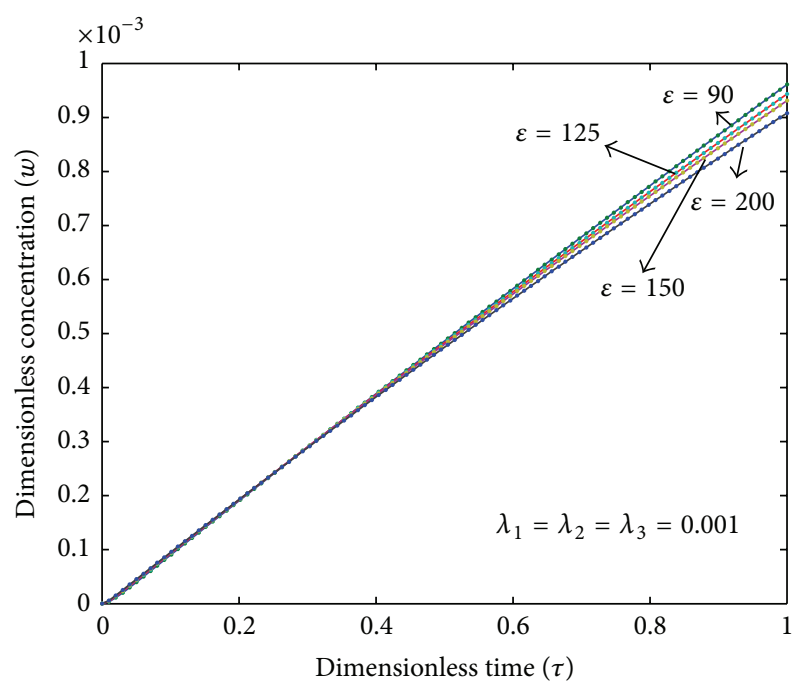

(a)

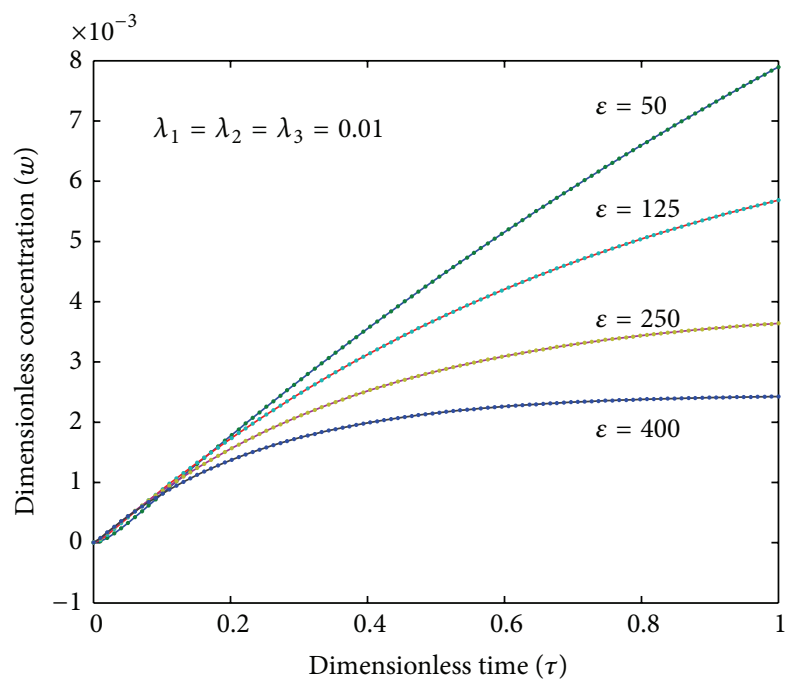

(b)

FIGURE 5: Profile of dimensionless free enzyme product concentration $w$ is calculated using (35) for various values of dimensionless parameters $\varepsilon, \lambda_{1}, \lambda_{2}$ and $\lambda_{3}$. Solid line: (35); dotted line: numerical simulation.

\section{Appendix}

\section{Numerical Simulation Program for (14)-(16)}

function graphmain3

options = odeset ("RelTol", $1 e-6$, "Stats", "on");

$\%$ initial conditions

$X 0=[1 ; 0 ; 0]$

$t$ span $=[0,5]$

tic

$[t, X]=$ ode45 (@TestFunction, $t$ span, $X 0$, options);

toc

figure

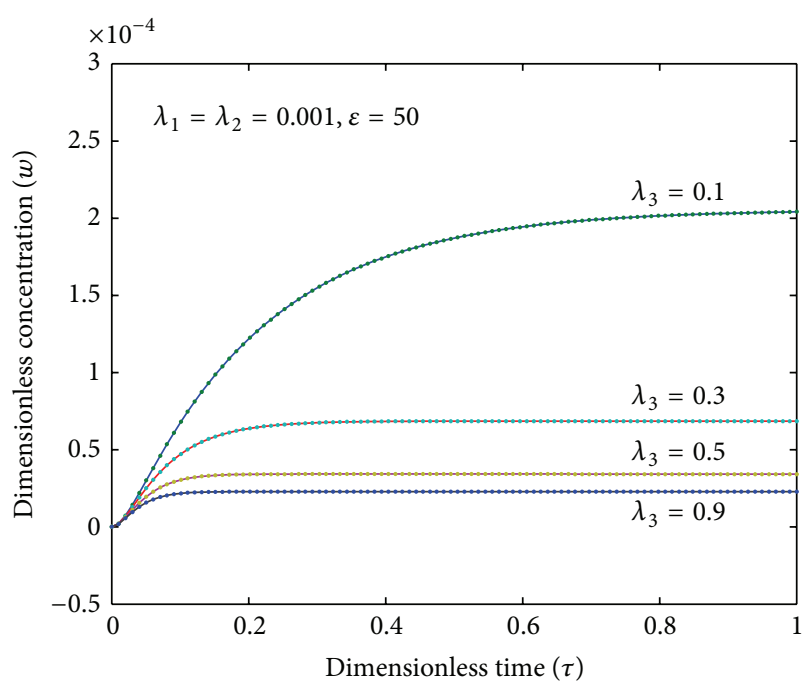

FIGURE 6: Profile of dimensionless free enzyme product concentration $v$ is calculated using (35) for various values of dimensionless parameter $\lambda_{3}$. Solid line: (35); dotted line: numerical simulation.

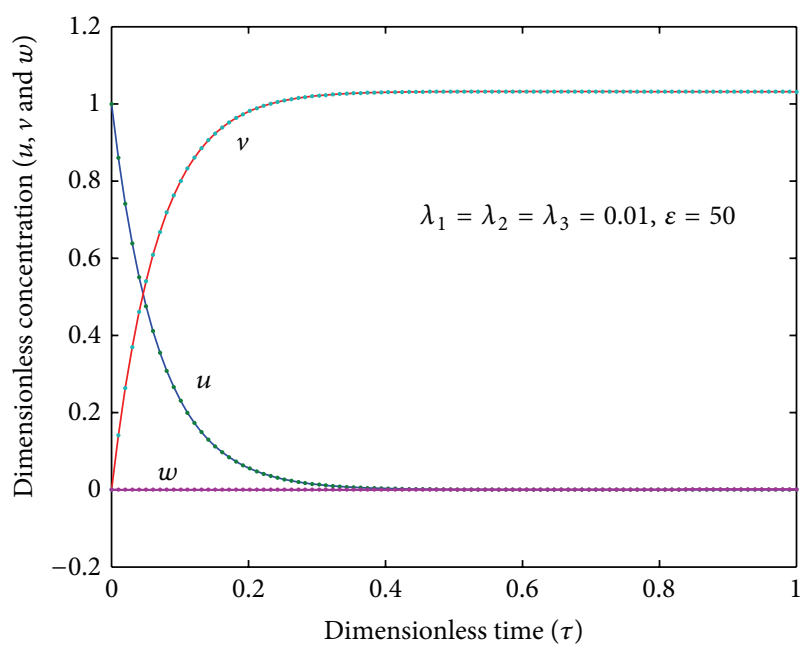

FIGURE 7: Profile of dimensionless concentrations $u$, $v$, and $w$ versus the dimensionless time $\tau$ using (33), (34), and (35) for the fixed values of the parameters $\lambda_{1}, \lambda_{2}, \lambda_{3}$, and $\varepsilon$. Solid line: (33), (34), and (35); dotted line: numerical simulation.

hold on

plot $(t, X(:, 1)$, ,-”)

plot $(t, X(:, 2)$, , “”)

plot $(t, X(:, 3), “ *$, $)$

legend (“ $x 1$ ”,“ $x 2$ ”,“ $x 3$ ”)

$y$ label (" $x$ ")

$x$ label (" $t$ ")

return

function $\left[d x_{-} d t\right]=$ TestFunction $(t, x)$

$e=1 ; c 1=0.1 ; c 2=0.001 ; c 3=0.1 ;$

$d x \_d t(1)=-e * x(1)+x(1) * x(2)+c 1 * x(2)$; 


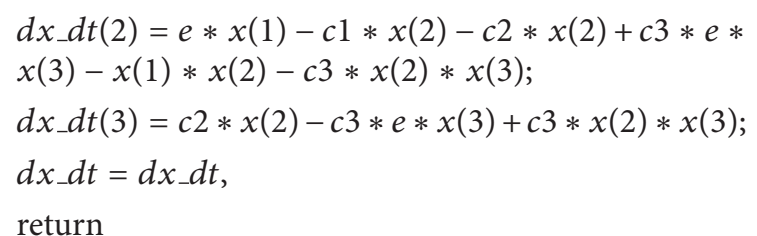

\section{Nomenclature and Units}

$\begin{array}{ll}{[\mathrm{E}]:} & \text { Enzyme concentration }(\mu \mathrm{M}) \\ {[\mathrm{C}]:} & \text { Enzyme-substrate complex }(\mu \mathrm{M}) \\ {[\mathrm{S}]:} & \text { Substrate concentration }(\mu \mathrm{M}) \\ {\left[\mathrm{E}_{0}\right]:} & \text { Initial enzyme concentration }(\mu \mathrm{M}) \\ K_{M}: & \text { Michaelis-Menten constant } \\ {\left[\mathrm{S}_{0}\right]:} & \text { Initial substrate concentration }(\mu \mathrm{M}) \\ k_{1}, k_{2}, k_{3}: & \text { Positive rate constants (none) } \\ \lambda_{1}, \lambda_{2}, \lambda_{3}, \varepsilon: & \text { Reaction diffusion parameter (none) } \\ u: & \text { Dimensionless substrate concentration } \\ & \text { (none) } \\ v: & \text { Dimensionless enzyme substrate } \\ w: & \text { concentration (none) } \\ & \text { Dimensionless product concentration } \\ t: & \text { (none) } \\ \tau: & \text { Time (sec) }\end{array}$

\section{Acknowledgment}

This work was supported by CSIR Project (no. 01 (2442)/ 10/EMR-II). The authors are thankful to the Principal and the Secretary, the Madura College, Madurai, for their encouragement.

\section{References}

[1] A. Beloqui, P. D. de María, P. N. Golyshin, and M. Ferrer, "Recent trends in industrial microbiology," Current Opinion in Microbiology, vol. 11, no. 3, pp. 240-248, 2008.

[2] B. M. Nestl, B. A. Nebel, and B. Hauer, "Recent progress in industrial biocatalysis," Current Opinion in Chemical Biology, vol. 15, no. 2, pp. 187-193, 2011.

[3] C. M. Clouthier and J. N. Pelletier, "Expanding the organic toolbox: a guide to integrating biocatalysis in synthesis," Chemical Society Reviews, vol. 41, no. 4, pp. 1585-1605, 2012.

[4] U. T. Bornscheuer, G. W. Huisman, R. J. Kazlauskas, S. Lutz, J. C. Moore, and K. Robins, "Engineering the third wave of biocatalysis," Nature, vol. 485, pp. 185-194, 2012.

[5] J. Keener and J. Sneyd, Mathematical Physiology, Springer, New York, NY, USA, 1998.

[6] M. E. G. Lyons, J. C. Greer, C. A. Fitzgerald, T. Bannon, and P. N. Barlett, "Reaction/diffusion with Michaelis-Menten kinetics in electroactive polymer films. Part 1 . The steady-state amperometric response," Analyst, vol. 121, no. 6, pp. 715-731, 1996.

[7] M. E. G. Lyons, T. Bannon, G. Hinds, and S. Rebouillat, "The home of premier fundamental discoveries, inventions and applications in the analytical and bioanalytical sciences," Analyst, vol. 123, p. 1947, 1998.

[8] L. Michaelis and M. Menten, "Die Kinetik der Invertinwirkung," Biochemische Zeitschrift, vol. 49, p. 333, 1913.
[9] A. Fersht, Enzyme Structure Mechanism, W.H. Freeman, New York, NY, USA, 2nd edition, 1985.

[10] K. M. Plowman, Enzyme Kinetics, McGraw-Hill, New York, NY, USA, 1972.

[11] J. Hurlebaus, A Pathway Modeling Tool for Metabolic Engineering, Institute für Biotechnologie Jülich-3912 Bundesrepublik Deutschland, 2001.

[12] J. D. Murray, Mathematical Biology, Springer, Berlin, Germany, 1989.

[13] J.-H. He, "Approximate analytical solution for seepage flow with fractional derivatives in porous media," Computer Methods in Applied Mechanics and Engineering, vol. 167, no. 1-2, pp. 57-68, 1998.

[14] J. H. He, "Application of homotopy perturbation method to nonlinear wave equations," Chaos, Solitons \& Fractals, vol. 26, no. 3, pp. 695-700, 2005.

[15] J.-H. He, "Homotopy perturbation method for solving boundary value problems," Physics Letters A, vol. 350, no. 1-2, pp. 8788, 2006.

[16] P. D. Ariel, "Homotopy perturbation method and the natural convection flow of a third grade fluid through a circular tube," Nonlinear Science Letters A, vol. 1, pp. 43-52, 2010.

[17] D. D. Ganji and M. Rafei, "Solitary wave solutions for a generalized Hirota-Satsuma coupled KdV equation by homotopy perturbation method," Physics Letters A, vol. 356, no. 2, pp. 131137, 2006

[18] A. Golbabai and B. Keramati, "Modified homotopy perturbation methodfor solving Fredholm integral equations," Chaos, Solitons \& Fractals, vol. 37, no. 5, pp. 1528-1537, 2008.

[19] M. Ghasemi, M. T. Kajani, and E. Babolian, "Numerical solutions of the nonlinear Volterra-Fredholm integral equations by using homotopy perturbation method," Applied Mathematics and Computation, vol. 188, no. 1, pp. 446-449, 2007.

[20] J. Biazar and H. Ghazvini, "He's homotopy perturbation method for solving systems of Volterra integral equations of the second kind," Chaos, Solitons \& Fractals, vol. 39, no. 2, pp. 770-777, 2009.

[21] Z. Odibat and S. Momani, "A reliable treatment of homotopy perturbation method for Klein-Gordon equations," Physics Letters A, vol. 365, no. 5-6, pp. 351-357, 2007.

[22] M. S. H. Chowdhury and I. Hashim, "Solutions of timedependent Emden-Fowler type equations by homotopyperturbation method," Physics Letters A, vol. 368, no. 3-4, pp. 305-313, 2007.

[23] J.-H. He, "Some asymptotic methods for strongly nonlinear equations," International Journal of Modern Physics B, vol. 20, no. 10, pp. 1141-1199, 2006. 

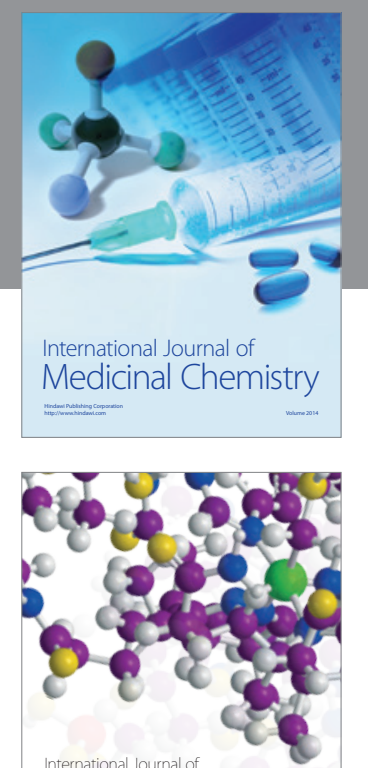

\section{Carbohydrate} Chemistry

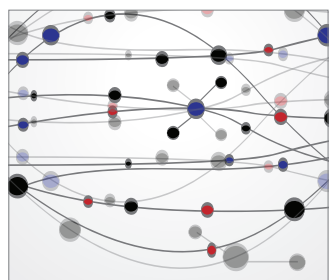

The Scientific World Journal
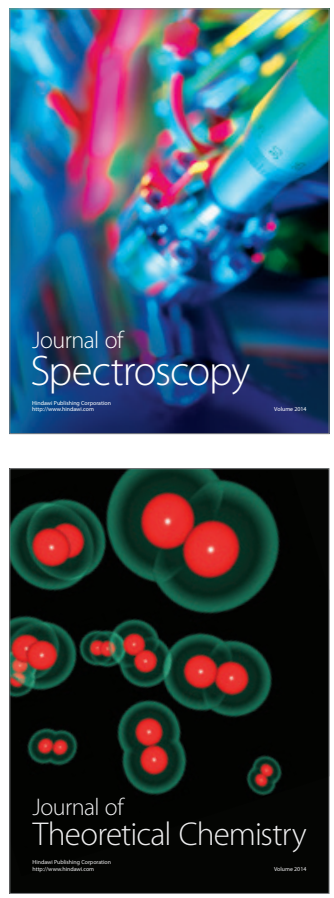
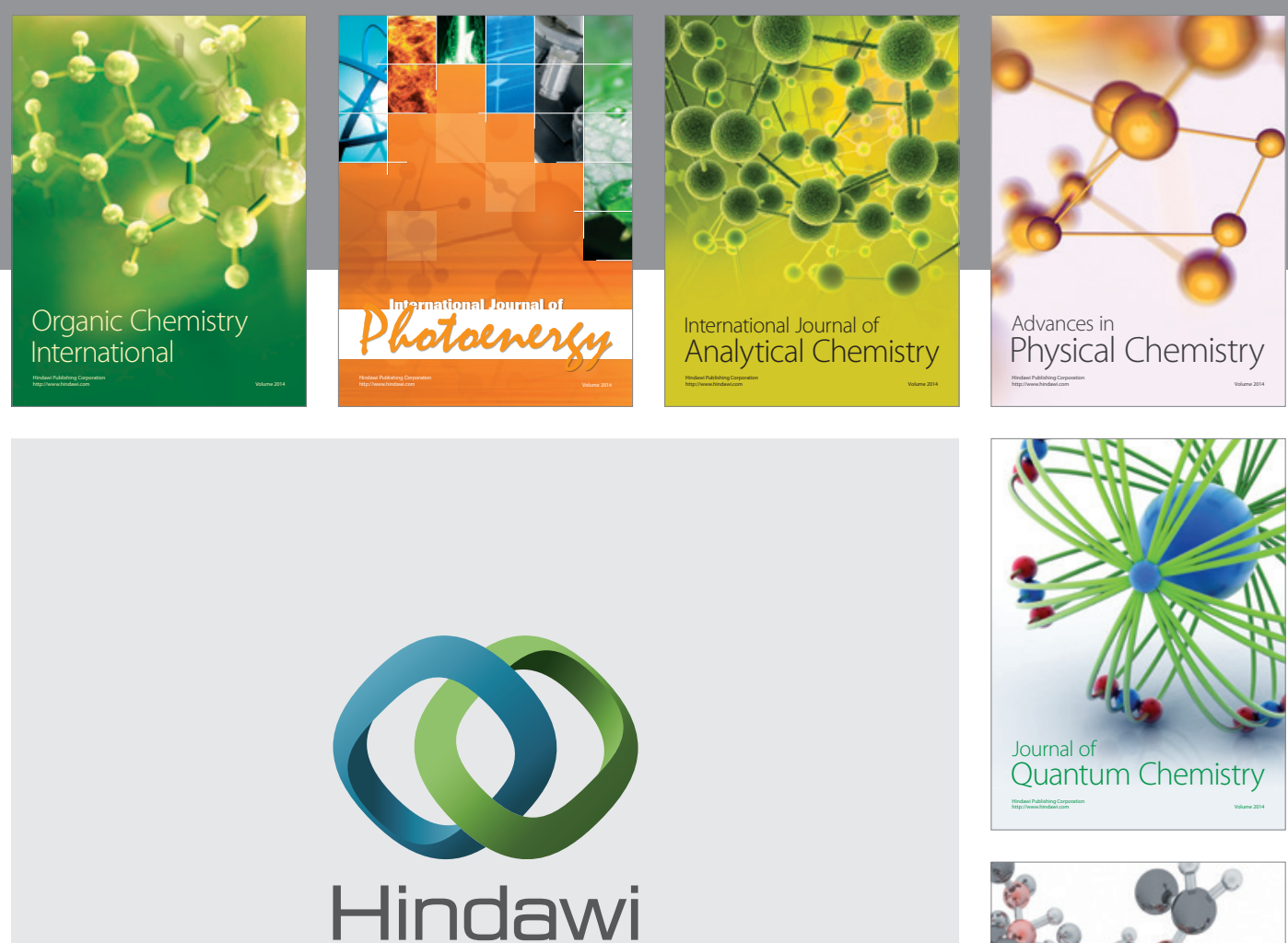

Submit your manuscripts at

http://www.hindawi.com

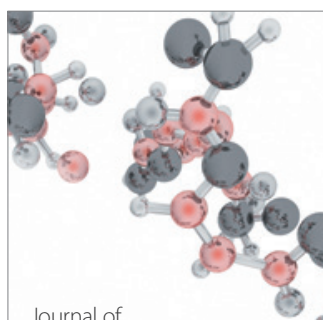

Analytical Methods

in Chemistry

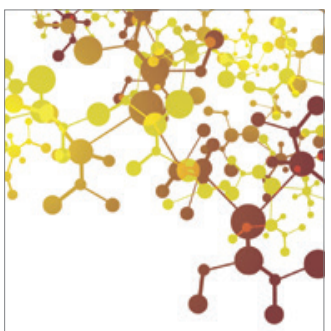

Journal of

Applied Chemistry

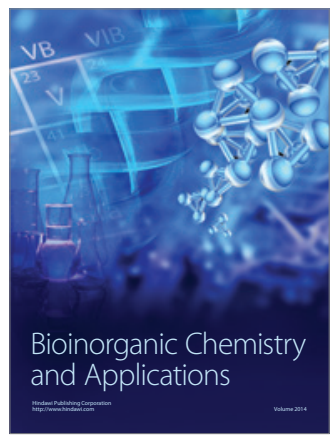

Inorganic Chemistry
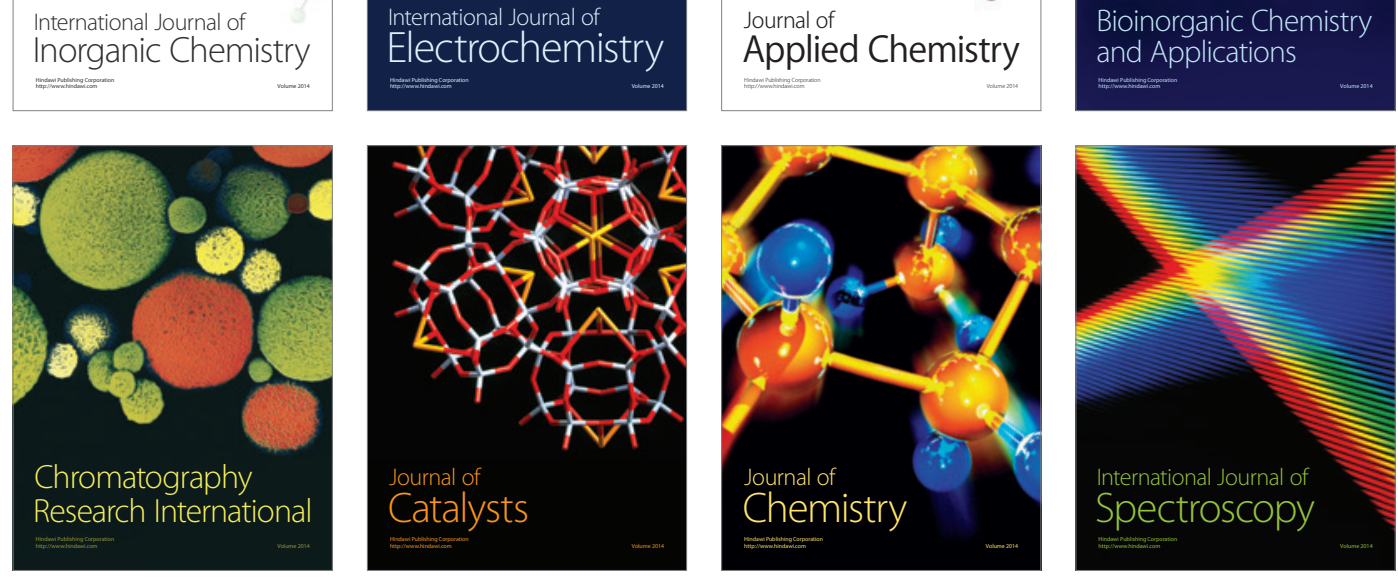